\title{
Huge right ventricle cardiac fibroma in a child patient
}

\author{
Qiang Fan ${ }^{1}$, Yunfei Ling ${ }^{1}$, and Qi An ${ }^{1}$ \\ ${ }^{1}$ Sichuan University West China Hospital
}

March 22, 2021

\begin{abstract}
In this case report, we describe a rare large right ventricular fibroma with sudden chest pain in a 9 year old child. The tumor was successfully surgical removed under cardiopulmonary bypass, but there was only a small remnant of the tumor to avoid rupture of the right ventricle and injury to the tricuspid valve. Pathological examination confirmed that the tumor was fibrous borderline tumor. The patient's heart function and the size of atrium and ventricles were normal, and there was no tricuspid regurgitation at 3-months follow-up. It was reported that the age less than 17 years old at time of diagnosis are associated with a poor prognosis, the long term outcome for this children patient needs further follow-up.
\end{abstract}

\section{Huge right ventricle cardiac fibroma in a child patient}

Qiang Fan*, Yunfei Ling*, Qi An\#

Department of Cardiovascular surgery, West China Hospital, Sichuan University

* These authors contributed equally to this work and should be considered co-first authors \#Corresponding author: Qi An

Address: No. 37 GuoXue Xiang, Chengdu, Sichuan, 610041, People's Republic of China

Tel: +862885422897

Fax: +862885422897

Email:anqi@scu.edu.cn

Conflicts of Interest: None

Funding: None.

Word count: 280

Key words cardiac fibroms, right ventricle, surgical treatment

Abstract In this case report, we describe a rare large right ventricular fibroma with sudden chest pain in a 9 year old child. The tumor was successfully surgical removed under cardiopulmonary bypass, but there was only a small remnant of the tumor to avoid rupture of the right ventricle and injury to the tricuspid valve. Pathological examination confirmed that the tumor was fibrous borderline tumor. The patient's heart function and the size of atrium and ventricles were normal, and there was no tricuspid regurgitation at 3-months follow-up. It was reported that the age less than 17 years old at time of diagnosis are associated with a poor prognosis, the long term outcome for this child patient needs further follow-up.

A previously healthy 9 -years-old boy presented to our hospital with a sudden chest pain for 2 weeks. There were no abnormities detected in physical examination. And electrocardiogram and chest X-ray was normal. Transthoracic echocardiography revealed a $45 \times 75 \mathrm{~mm}$ well-mass in the lateral and anterior wall of right 
ventricle adjacent to right atrioventricular groove and compressing right heart chamber (panel A, arrow; Supplementary material online, Video 1). Cardiac magnetic resonance (CMR) LGE imaging in short-axis view showed inhomogeneous delayed enhancement (panel B, arrow) and CMR perfusion imaging in four-chamber view showed very little blood perfusion (panel C, arrow) of the mass. Volume-rendered image of the heart from different perspectives showed exactly the position and surrounding structures of the mass (Supplementary material online, Video 2). Considering the tumor size and patient's symptom, surgical resection through a median sternotomy under cardiopulmonary bypass. The tumor has an intact adventitia extending from the epicardium (Supplementary material online, Video 3). To avoid the rupture of right ventricle and injury to tricuspid valve, the tumor was removed nearly completely (panel D and E). The cavity left by excision of the tumor was filled by in-situ suture of the residual tissue. Intraoperative transoesophageal echocardiography showed there was mild tricuspid regurgitation and good heart function. The pathologic diagnosis was mesenchymal and fibrogenic tumor that prone to ligamentoid fibromatosis (panel F). Immunohistochemistry is positive for smooth muscle actin (panel G), whereas CD34, S100 are negative that confirmed the fibrous origin of the tumor. The patient recovered well and discharged unevenfully. The patient's heart function and the size of atrium and ventricles were normal, and there was no tricuspid regurgitation at 3-months follow-up.

Consent The study were approved by the relevant ethics committees, and oral informed consent was obtained for the participant.

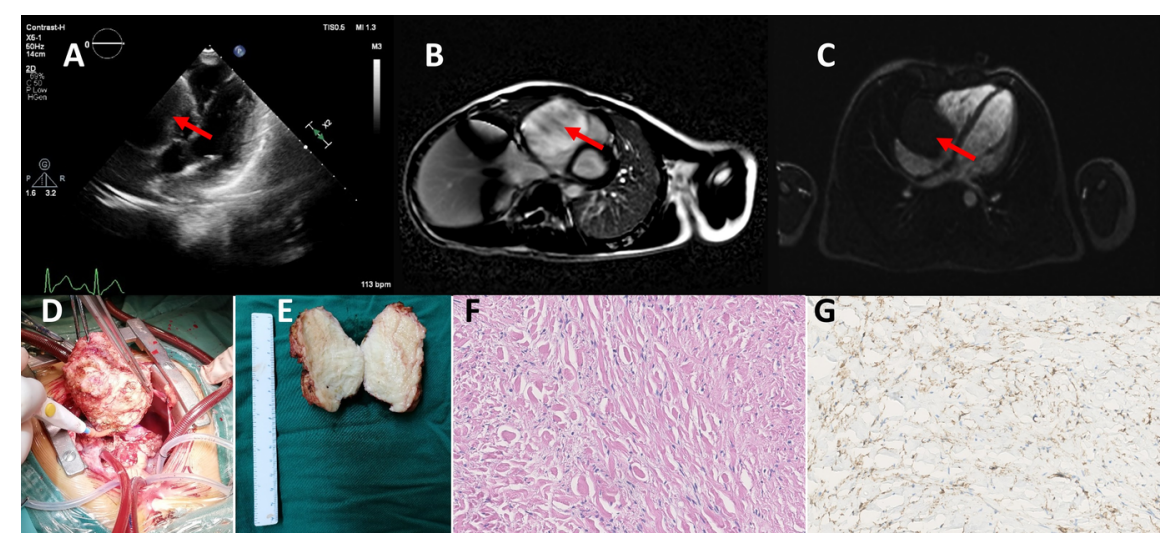

